\title{
Power-law corrections to entanglement entropy of horizons
}

\author{
Saurya Das, ${ }^{1, *}$ S. Shankaranarayanan, ${ }^{2, \dagger}$ and Sourav Sur ${ }^{1, \ddagger}$ \\ ${ }^{1}$ Department of Physics, University of Lethbridge, 4401 University Drive, Lethbridge, Alberta, Canada T1K $3 M 4$ \\ ${ }^{2}$ Max-Planck-Institut für Gravitationphysik, Am Mühlenberg 1, D-14476 Potsdam, Germany
}

(Received 29 December 2007; published 10 March 2008)

\begin{abstract}
We reexamine the idea that the origin of black-hole entropy may lie in the entanglement of quantum fields between the inside and outside of the horizon. Motivated by the observation that certain modes of gravitational fluctuations in a black-hole background behave as scalar fields, we compute the entanglement entropy of such a field, by tracing over its degrees of freedom inside a sphere. We show that while this entropy is proportional to the area of the sphere when the field is in its ground state, a correction term proportional to a fractional power of area results when the field is in a superposition of ground and excited states. The area law is thus recovered for large areas. Further, we identify the location of the degrees of freedom that give rise to the above entropy.
\end{abstract}

DOI: 10.1103/PhysRevD.77.064013

\section{INTRODUCTION}

Almost four decades ago, Bekenstein argued that if the second law of thermodynamics is not to be violated in the presence of a black hole, the black hole must possess an entropy proportional to its horizon area [1]. The significance of this result became clear with Hawking's demonstration of black-hole thermal radiation [2]. Hawking showed that quantum effects in the background of a body collapsing to a Schwarzschild black hole (BH) of mass $M$, will lead, at late times, to a radiation of particles in all modes of the quantum field, with a characteristic thermal spectrum at a temperature equal to

$$
T_{\mathrm{H}}=\left(\frac{\hbar c}{k_{B}}\right) \frac{\kappa}{2 \pi}=\left(\frac{\hbar c^{3}}{G k_{B}}\right) \frac{1}{8 \pi M},
$$

where $\kappa$ is the surface gravity of the black-hole, $G$ is the four-dimensional Newton's constant, and $k_{B}$ is the Boltzmann constant. Since the Hawking temperature fixes the factor of proportionality between temperature and surface gravity, one finds the Bekenstein-Hawking area law

$$
S_{\mathrm{BH}}=\left(\frac{k_{B}}{4}\right) \frac{\mathcal{A}_{\mathrm{H}}}{\ell_{\mathrm{Pl}}^{2}} \quad \text { where } \ell_{\mathrm{Pl}}=\sqrt{\frac{G \hbar}{c^{3}}},
$$

$\mathcal{A}_{\mathrm{H}}$ is the horizon area, and $\ell_{\mathrm{Pl}}$ is the four-dimensional Planck length. All known black holes in $n(>2)$ space-time dimensions satisfy the area law (AL).

The universality of the AL has raised some important questions that remain unanswered: what is the dynamical mechanism that makes $S_{\mathrm{BH}}$ a universal function, independent of the black hole's past history and detailed internal condition? Why is $S_{\mathrm{BH}}$ proportional to $\mathcal{A}_{\mathrm{H}}$ ? What is the microscopic origin of BH entropy? Are there corrections to the entropy and if so, how generic are these corrections?

\footnotetext{
*saurya.das@uleth.ca

†shanki@aei.mpg.de

"sourav.sur@uleth.ca
}

PACS numbers: 04.60. $-\mathrm{m}, 04.70 .-\mathrm{s}, 04.70 . D y, 03.65 . U d$

Where are the degrees of freedom, responsible for the entropy, located? These questions often seem related, which a correct theory of quantum gravity is expected to address.

Naturally, there has been considerable work attempting to address some of the above questions (for recent reviews, see Refs. $[3,4])$. Broadly, there have been two approaches: (i) associating $S_{\mathrm{BH}}$ with fundamental degrees of freedom of a microscopic theory of quantum gravity [5] and (ii) associating $S_{\mathrm{BH}}$ with quantum matter fields propagating in a fixed $\mathrm{BH}$ background [6-8].

In this work, we focus on the second approach and, in particular, we attribute $S_{\mathrm{BH}}$ to the entanglement of the quantum fields inside and outside the horizon. We show that it is possible to: (a) obtain generic power-law corrections to Eq. (2) which are small for large horizon radii, can become significant for small horizons, (b) identify the degrees of freedom that give rise to entanglement entropy, and (c) test the robustness of Eq. (2) and its corrections for massive quantum fields.

Consider a quantum scalar field (in a pure state) propagating in the $\mathrm{BH}$ space-time. For an outside observer, the $\mathrm{BH}$ horizon provides a boundary and (s)he can only have information of the state restricted to outside the horizon. Consequently, while the full state of the field is pure, the state restricted to outside the horizon is mixed which leads to a nonzero entropy. This entropy, aka Von Neumann entropy, can formally be written as

$$
S=-k_{B} \operatorname{Tr}(\rho \ln \rho)
$$

where $\rho$ is the mixed (or reduced) density matrix. The above microcanonical definition of entropy will be used here. Although it is also possible to compute the entanglement entropy in the canonical picture [8], its usage is restrictive due to the fact that it implicitly assumes positive specific heat. On the contrary, however, a Schwarzschild $\mathrm{BH}$ has a negative specific heat.

About two decades ago, Bombelli et al [6] showed that the entanglement entropy of scalar fields is proportional to 
$\mathcal{A}_{H} / a^{2}$, where $\mathcal{A}_{H}$ is the area of the boundary of the region being traced over (the "horizon"), and $a$ is the ultraviolet cutoff at the horizon (equivalently, the lattice spacing, when space is discretized). Identifying $a$ with the Planck length $\ell_{\mathrm{Pl}}$, one recovers the Bekenstein-Hawking AL (2). These results were later reproduced by Srednicki [7], where by tracing over the degrees of freedom inside a spherical surface of radius $R$, he showed that the entanglement entropy

$$
S \sim \frac{\mathcal{A}}{a^{2}},
$$

where $\mathcal{A}=4 \pi R^{2}$. Thus, the area law can be considered as a consequence of the entanglement of the quantum fields across the horizon. ${ }^{1}$ (An analytical proof of the area proportionality has recently been given in Ref. [10]. For an application of entanglement in stringy black holes, see Refs. [11,12].)

Now, why is such a computation involving scalar fields in flat space-time relevant for black holes at all? Here, we try to provide at least a plausible answer to this question: in Appendix A, we consider gravitational perturbations in a black-hole background, and show that for certain modes of these perturbations, the effective action reduces to that of a scalar field. Further, in Appendix B, we write the corresponding scalar field Hamiltonian in the black-hole background in Lemaitre coordinates, and show that for a fixed Lemaitre time, it reduces to that in a flat space-time. Thus, for time-independent quantities such as entropy, scalar fields of the type considered here appear to play an important role.

The computation and the area proportionality of entanglement entropy in [6] and Srednicki [7] is based on the simplifying assumption that the quantum field is in the vacuum (i.e., ground) state (GS). Recently, two of the authors (S.D. and S. Sh.) investigated the robustness of the entanglement entropy: area-law relation by considering nonvacuum states $[13,14]$. It was shown that while replacing the vacuum state by a generic coherent state (GCS) or a class of squeezed states (SS) does not affect the AL, doing so with a class of $n$-particle or excited states (ES) results in a significant deviation from the AL. More specifically, if the scalar field is in a class of 1-particle ES, it was shown that the entropy scales as

$$
S \sim\left(\frac{\mathcal{A}}{a^{2}}\right)^{\mu},
$$

where the power $\mu$ is always less than unity, and decreases with the increase in the number of excitations [14] (see Appendix $\mathrm{C}$ for details). Thus, it was shown that the

\footnotetext{
${ }^{1}$ Although one recovers the area law, the divergence of the entanglement entropy has been a puzzle. The reason for the divergence is due to the fact that the boundary delineating the region being traced over is sharp [9]
}

entanglement entropy does not always lead to AL and its form crucially depends on the choice of the quantum state.

Given the above results, one may draw two distinct conclusions: first that entanglement entropy is not robust-and reject it as a possible source of BH entropy. Second-since entanglement entropy for ES scales as a lower power of area-it is plausible that when a generic state (consisting of a superposition of GS and ES) is considered, corrections to the Bekenstein-Hawking entropy will emerge. In order to determine which one is correct, it is imperative to investigate various generalizations of the scenarios considered in Refs. [6,7,14]. To this end, in this work we calculate the entanglement entropy of the mixed superposition of vacuum and 1-particle state (MS). We show explicitly that the MS entanglement entropy is given by

$$
S=c_{0}\left(\frac{\mathcal{A}}{a^{2}}\right)\left[1+c_{1} f(\mathcal{A})\right] \quad \text { where } f(\mathcal{A})=\left(\frac{\mathcal{A}}{a^{2}}\right)^{-\nu},
$$

$c_{0}, c_{1}$ are constants of order unity, and $\nu$ is a fractional power which depends on the amount of mixing. Thus, we show that, for large horizon area $\left(\mathcal{A} \gg a^{2}\right)$, the contribution of $f(\mathcal{A})$ is negligible and the MS entanglement entropy asymptotically approaches the GS entropy. This is significantly different from the 1-particle state considered in Ref. [14] for which the entropy always scales as power of area, cf. Eq. (5), the power being less than unity. Thus, if black-hole entropy is a consequence of quantum entanglement, the AL is valid for large horizons, as long as the quantum field is in a superposition of vacuum and 1particle states.

From a physics point of view, we expect power-law corrections to Bekenstein-Hawking entropy for the following two reasons: (a) $S_{\mathrm{BH}}$ is a semiclassical result and is valid for large black holes, i.e. when $r_{h} \gg \ell_{\mathrm{Pl}}\left(r_{h}\right.$ is the radius of the horizon). It is not clear whether the AL will be valid for the small BHs $\left(r_{h} \sim \ell_{\mathrm{Pl}}\right)$. (b) There is no reason to expect that the Bekenstein-Hawking entropy to be the whole answer for a correct theory of quantum gravity. For instance, it was shown by Wald [15] that if one takes into account higher curvature corrections to the EinsteinHilbert action, the Bekenstein-Hawking entropy is the leading term in a series expansion.

As mentioned earlier, in this paper, we also identify the precise location of the microscopic degrees of freedom (DOF) for the entanglement entropy of the superposition of vacuum and 1-particle states [16]. We find that the DOF close to the horizon contribute most to the total entropy. However, there are small contributions from the DOF far away from the horizon as well. These far-away DOF contributions are least in the case of vacuum state and increase as the number of excitations and/or the mixing weight of 1particle state with vacuum state increases. Correspondingly, deviations from the AL increases as well. Thus, 
the corrections to the AL may, in a way, be attributed to the far-away DOF.

Finally, we investigate the effect of massive fields on entanglement entropy. We show that in all cases (vacuum, 1-particle, and superposed states), the massive scalar field entanglement entropy $\left(S_{m}\right)$ contains an exponential damping factor in comparison with the massless scalar field entanglement entropy $\left(S_{0}\right)$. The Gaussian fits of the ratio $S_{m} / S_{0}$ with the mass (in appropriate units) show that the exponential factor depends explicitly on the mass squared and hence falls off rapidly as the mass is increased. However, there is not much variation of the fitting parameters for the different-vacuum, 1-particle, and mixedstates, even when a fairly high amount of excitation is taken into account. This shows that the mixing proportions in the GS and ES superposition have little influence on the ratio $S_{m} / S_{0}$. We thus show that the mass overall reduces the entropy exponentially.

The organization of this paper is as follows: In the next section, we briefly review the procedure of obtaining the entanglement entropy of massless scalar fields in flat space-time. In Sec. III, we obtain the (reduced) density matrix for the scalar field which is in a superposition of GS and 1-particle ES. We compute the entanglement entropy numerically for such a superposition and estimate the corrections to the BH area law. In Sec. IV, we identify the locations of the scalar field degrees of freedom that are responsible for the entanglement entropy for the superposition of GS and ES. In Sec. V, we obtain the entanglement entropy for a massive scalar field. We conclude with a summary and open questions in Sec. VI. In Appendix A, we discuss the motivation for considering a massless or massive scalar field for computing the entanglement entropy, and as mentioned before, we show that for certain modes of gravitational perturbations, the relevant action reduces to that of a scalar field. In Appendix B, we obtain the Hamiltonian of a scalar field in a general $\mathrm{BH}$ spacetime. We show that this Hamiltonian in Lemaitre coordinates, and at a fixed Lemaitre time, reduces to the scalar field Hamiltonian in flat space-time. Thus this Hamiltonian is relevant for the computation of time-independent quantities such as entropy. In Appendix $C$ we briefly review the results obtained in the earlier works $[6,7,14]$ for the ground state and 1-particle state.

Before we proceed, a few comments on the notation we use are in order: The metric is four-dimensional with the signature $(-,+,+,+)$. We use units with $k_{B}=c=\hbar=1$ and set $M_{\mathrm{Pl}}^{2}=1 /(16 \pi G)$. The quantum field $\varphi$ is a minimally coupled scalar field.

\section{ENTANGLEMENT ENTROPY OF SCALAR FIELDS}

In this section, we briefly review the procedure of obtaining entanglement entropy for scalar fields propagating in flat space-time. The motivation for considering scalar fields for the entanglement entropy computations is discussed in Appendix A. The relevance of the scalar field Hamiltonian in flat space-time for computing entropy in a black-hole space-time is discussed in Appendix B.

The Hamiltonian of a massless scalar field propagating in flat space-time is given by Eq. (B15). In order to obtain the entropy, we need to discretize this Hamiltonian on the radial lattice with lattice spacing $a$. Discretizing the Hamiltonian such that $r \rightarrow r_{i} ; r_{i+1}-r_{i}=a$ and $L=$ $(N+1) a$ is the infrared cutoff, ${ }^{2}$ we get

$$
\begin{aligned}
H_{l m}= & \frac{1}{2 a} \sum_{j=1}^{N}\left[\pi_{l m, j}^{2}+\left(j+\frac{1}{2}\right)^{2}\left(\frac{\varphi_{l m, j}}{j}-\frac{\varphi_{l m, j+1}}{j+1}\right)^{2}\right. \\
& \left.+\frac{l(l+1)}{j^{2}} \varphi_{l m, j}^{2}\right], \\
H= & \sum_{l m} H_{l m},
\end{aligned}
$$

where $\quad \varphi_{l m, j} \equiv \varphi_{l m}\left(r_{j}\right), \pi_{l m} \equiv \pi_{l m, j}\left(r_{j}\right) \quad$ and $\left[\varphi_{l m, j}, \pi_{l^{\prime} m^{\prime}, j^{\prime}}\right]=i \delta_{l l^{\prime}} \delta_{m m^{\prime}} \delta_{j j^{\prime}}$. Up to the overall factor of $a^{-1}$, Eq. (7) is identical to the Hamiltonian of $N$ coupled harmonic oscillators (HOs):

$$
H=\frac{1}{2} \sum_{i=1}^{N} p_{i}^{2}+\frac{1}{2} \sum_{i, j=1}^{N} x_{i} K_{i j} x_{j}
$$

where the matrix $K_{i j}$ represents the potential energy and the interaction between the oscillators $(i, j=1, \ldots, N$, the coordinates $x_{i}$ replace the field variables $\left.\varphi_{l m}\right)$. For the Hamiltonian (7), it is given by

$$
\begin{aligned}
K_{i j}= & \frac{1}{i^{2}}\left[l(l+1) \delta_{i j}+\frac{9}{4} \delta_{i 1} \delta_{j 1}+\left(N-\frac{1}{2}\right)^{2} \delta_{i N} \delta_{j N}\right. \\
& \left.+\left\{\left(i+\frac{1}{2}\right)^{2}+\left(i-\frac{1}{2}\right)^{2}\right\} \delta_{i, j(i \neq 1, N)}\right] \\
& -\left[\frac{\left(j+\frac{1}{2}\right)^{2}}{j(j+1)}\right] \delta_{i, j+1}-\left[\frac{\left(i+\frac{1}{2}\right)^{2}}{i(i+1)}\right] \delta_{i, j-1} .
\end{aligned}
$$

The last two terms denote nearest-neighbor interactions and originate from the derivative term in Eq. (B15). The most general eigenstate of the Hamiltonian (8) is a product of $N \mathrm{HO}$ wave functions:

$$
\psi\left(x_{1}, \ldots, x_{N}\right)=\prod_{i=1}^{N} \mathcal{N}_{i} \mathcal{H}_{\nu_{i}}\left(k_{D i}^{1 / 4} x_{i} \underline{\mathrm{x}}_{i}\right) \exp \left(-\frac{1}{2} k_{D i}^{1 / 2} \underline{\mathrm{x}}_{i}^{2}\right),
$$

where $\mathcal{N}_{i} \mathrm{~s}$ are the normalization constants given by

$$
\mathcal{N}_{i}=\frac{k_{D i}^{1 / 4}}{\pi^{1 / 4} \sqrt{2^{\nu_{i}} \nu_{i} !}}
$$

\footnotetext{
${ }^{2}$ In discretizing the terms containing the derivatives, one usually adopts the middle-point prescription, i.e., the derivative of the form $f(x) d_{x}[g(x)]$ is replaced by $f_{j+1 / 2}\left[g_{j+1}-g_{j}\right] / a$.
} 
$\underline{\mathrm{x}}=U x \quad\left(U^{T} U=I_{N}\right), \quad x^{T}=\left(x_{1}, \ldots, x_{N}\right), \quad \underline{\mathrm{x}}^{T}=$ $\left(\underline{\mathrm{x}}_{1}, \ldots, \underline{\mathrm{x}}_{N}\right), K_{D} \equiv U K U^{T}$ is a diagonal matrix with elements $k_{D i}$, and $\nu_{i}(i=1 \ldots N)$ are the indices of the Hermite polynomials $\left(\mathcal{H}_{\nu}\right)$. The frequencies are ordered such that $k_{D i}>k_{D j}$ for $i>j$.

Defining the $N \times N$ matrix $\Omega=U^{T} K_{D}^{1 / 2} U$, such that $|\Omega|=\left|K_{D}\right|^{1 / 2}$, and tracing over the first $n$ of the $N$ oscillators, one obtains the reduced density matrix:

$$
\begin{aligned}
\rho\left(t ; t^{\prime}\right)= & \int \prod_{i=1}^{n} d x_{i} \psi\left(x_{1}, \ldots, x_{n} ; t\right) \psi^{\star}\left(x_{1}, \ldots, x_{n} ; t^{\prime}\right) \\
= & \int \prod_{i=1}^{n} d x_{i} \exp \left[-\frac{x^{T} \Omega x}{2}\right] \prod_{i=1}^{N} \mathcal{N}_{i} \mathcal{H}_{\nu_{i}}\left(k_{D i}^{1 / 4} \underline{\mathbf{x}}_{i}\right) \\
& \times \exp \left[-\frac{x^{\prime T} \Omega x^{\prime}}{2}\right] \prod_{j=1}^{N} \mathcal{N}_{j} \mathcal{H}_{\nu_{j}}\left(k_{D i}^{1 / 4} \underline{\mathbf{x}}_{i}^{\prime}\right)
\end{aligned}
$$

where we now denote $x^{T}=\left(x_{1}, \ldots, x_{n} ; t_{1}, \ldots, t_{N-1}\right)=$ $\left(x_{1}, \ldots, x_{n} ; t\right), \quad$ with $\quad t \equiv t_{1}, \ldots, t_{N-n} ; t_{j} \equiv x_{n+j}, j=$ $1, \ldots,(N-n)$. It is easy to check that $\rho^{2} \neq \rho$, implying that $\rho$ is mixed, i.e., although the full state is pure, the state obtained by integrating over $n \mathrm{HO}$ is mixed. Substituting the reduced density matrix (12) into the formal expression (3) will yield a nonzero (positive) entanglement entropy.

It is not possible to obtain a closed form expression for the density matrix for an arbitrary state (10). However, in the cases where all the HOs are in their GS [7], or in the GCS or in a class of SS [14], all of which are minimum uncertainty states, closed form analytic expressions of $\rho\left(t ; t^{\prime}\right)$, and hence of the entropy, can be evaluated exactly and shown to follow the BH AL. For the first ES, not a minimum uncertainty state, the entropy computed numerically [14] is found not to obey the AL.

In the following section, we obtain the entanglement entropy for the superposition of GS and ES. (For the sake of completeness, we have briefly discussed the entanglement entropy for the ground and first-excited states in the Appendix C.)

\section{ENTANGLEMENT ENTROPY FOR A SUPERPOSITION OF GS AND ES}

In this section, we obtain the entanglement entropy for the superposition of ground and excited states. (In the following, we denote all relevant quantities such as the wave function, density matrix, etc. by the symbol/suffix 0 for GS and by 1 for the first ES.)

The discretized scalar field wave function $\psi$ in an MS is a linear superposition of the N-HO GS wave function $\psi_{0}$, Eq. (C1) (Appendix C), and N-HO (1-particle) ES wave function $\psi_{1}$ [corresponding to one $\mathrm{HO}$ in the ES, while the rest $N-1$ in their GS, Eq. (C12)], i. e.,

$$
\psi(\hat{x} ; t)=\left[c_{0} \psi_{0}(\hat{x} ; t)+c_{1} \psi_{1}(\hat{x} ; t)\right]
$$

where $\hat{x} \equiv\left\{x_{1}, \cdots, x_{n}\right\} ;$ and as before $t_{j} \equiv x_{n+j}(j=$
$1, \cdots, N-n) ; t \equiv\left\{t_{1}, \cdots, t_{N-n}\right\}=\left\{x_{n+1}, \cdots, x_{N}\right\}$.

Normalization of $\psi$ requires $c_{0}^{2}+c_{1}^{2}=1$. Here we assume that $c_{0}$ and $c_{1}$ are real constants.

Referring to the Appendix C and using Eq. (C12), we can write

$$
\psi(\hat{x} ; t)=\left[c_{0}+c_{1} f(\hat{x} ; t)\right] \psi_{0}(\hat{x} ; t),
$$

where

$$
f(\hat{x} ; t)=\sqrt{2} \alpha^{T} K_{D}^{1 / 4} U x=y^{T} x,
$$

$\alpha$ being the expansion coefficient defined in Eq. (C14). The $N$-dimensional column vector $y$ is given by

$$
y=\sqrt{2} U^{T} K_{D}^{1 / 4} \alpha=\left(\begin{array}{l}
y_{A} \\
y_{B}
\end{array}\right) .
$$

$y_{A}$ and $y_{B}$ are $n$ - and $(N-n)$-dimensional column vectors, respectively.

The density matrix is a sum of three terms:

$$
\begin{aligned}
\rho\left(t ; t^{\prime}\right) & =\int \prod_{i=1}^{n} d x_{i} \psi(\hat{x} ; t) \psi^{\star}\left(\hat{x} ; t^{\prime}\right) \\
& =c_{0}^{2} \rho_{0}\left(t ; t^{\prime}\right)+c_{1}^{2} \rho_{1}\left(t ; t^{\prime}\right)+c_{0} c_{1} \rho_{2}\left(t ; t^{\prime}\right)
\end{aligned}
$$

where $\rho_{0}\left(t ; t^{\prime}\right)$ is the GS density matrix (C4) and $\rho_{1}\left(t ; t^{\prime}\right)$ is the ES density matrix (C15). It is easy to see that one can make the following identifications of the matrix $\Lambda$ and its components; and the constant $\kappa$ (see Appendix C), with the column vector $y$ and its components as

$$
\begin{gathered}
\Lambda=\frac{1}{2} y y^{T}=\left(\begin{array}{cc}
\Lambda_{A} & \Lambda_{B} \\
\Lambda_{B}^{T} & \Lambda_{C}
\end{array}\right), \quad \Lambda_{A}=\frac{1}{2} y_{A} y_{A}^{T}, \\
\Lambda_{B}=\frac{1}{2} y_{A} y_{B}^{T}, \quad \Lambda_{C}=\frac{1}{2} y_{B} y_{B}^{T}, \\
\kappa=\operatorname{Tr}\left(\Lambda_{A} A^{-1}\right)=\frac{1}{2} y_{A}^{T} A^{-1} y_{A} .
\end{gathered}
$$

$\rho_{2}$ is the cross term in the total density matrix $\rho$, Eq. (17), due to the mixing of GS and ES and can be evaluated as follows:

$$
\begin{aligned}
\rho_{2}\left(t ; t^{\prime}\right) & =\int \prod_{i=1}^{n} d x_{i}\left[f(\hat{x} ; t)+f\left(\hat{x} ; t^{\prime}\right)\right] \psi_{0}(\hat{x} ; t) \psi_{0}^{\star}\left(\hat{x} ; t^{\prime}\right) \\
& =\left(y_{B}-p\right)^{T}\left(t+t^{\prime}\right) \rho_{0}\left(t ; t^{\prime}\right),
\end{aligned}
$$

where

$$
p=B^{T} A^{-1} y_{A}
$$

is an $(N-n)$-dimensional column vector. ${ }^{3}$

Using Eqs. (C4), (C15), and (C19), the complete MS density matrix can be written as

\footnotetext{
${ }^{3}$ For definitions of matrices $A, B$, etc., see Appendix $\mathrm{C}$ [Eq. (C2)].
} 


$$
\rho\left(t ; t^{\prime}\right)=\left[c_{0}^{2}+c_{1}^{2} \kappa\left\{1+u\left(t ; t^{\prime}\right)\right\}+c_{0} c_{1} v\left(t ; t^{\prime}\right)\right] \rho_{0}\left(t ; t^{\prime}\right)
$$

where the functions $u$ and $v$ are defined by

$$
\begin{aligned}
& u\left(t ; t^{\prime}\right)=-\frac{t^{T} \Lambda_{\gamma} t+t^{\prime T} \Lambda_{\gamma} t^{\prime}}{2}+t^{T} \Lambda_{\beta} t^{\prime}, \\
& v\left(t ; t^{\prime}\right)=\left(y_{B}-p\right)^{T}\left(t+t^{\prime}\right) .
\end{aligned}
$$

Let us define

$$
F\left(t ; t^{\prime}\right)=1+\kappa_{1} w\left(t ; t^{\prime}\right)+\kappa_{2} v\left(t ; t^{\prime}\right)+\frac{\kappa_{2}^{2}}{2} v^{2}\left(t ; t^{\prime}\right)
$$

where

$$
\begin{aligned}
w\left(t ; t^{\prime}\right) & =-\frac{t^{T} \Lambda_{\gamma^{\prime}} t+t^{\prime T} \Lambda_{\gamma^{\prime}} t^{\prime}}{2}+t^{T} \Lambda_{\beta^{\prime}} t^{\prime}, \\
\Lambda_{\beta^{\prime}} & =\Lambda_{\beta}-2 \kappa_{0}\left(\Lambda_{\beta}-\frac{\Lambda_{C}}{\kappa}\right), \\
\Lambda_{\gamma^{\prime}} & =\Lambda_{\gamma}+2 \kappa_{0}\left(\Lambda_{\beta}-\frac{\Lambda_{C}}{\kappa}\right)
\end{aligned}
$$

and

$$
\begin{array}{cc}
\kappa_{0}=\frac{c_{0}^{2}}{\tilde{\kappa}}, & \kappa_{1}=\frac{c_{1}^{2}}{\tilde{\kappa}}, \\
\kappa_{2}=\frac{c_{0} c_{1}}{\tilde{\kappa}}, & \tilde{\kappa}=c_{0}^{2}+c_{1}^{2} \kappa .
\end{array}
$$

$\Lambda_{\beta^{\prime}}$ and $\Lambda_{\gamma^{\prime}}$ are $(N-n) \times(N-n)$ matrices, and constants $\left(\kappa_{0}, \kappa_{1}, \kappa_{2}\right)$ describe the amount of mixing between the GS and ES.

With these definitions, the density matrix (21) can be rewritten as

$$
\rho\left(t ; t^{\prime}\right)=\tilde{\kappa} F\left(t ; t^{\prime}\right) \rho_{0}\left(t ; t^{\prime}\right) .
$$

As for the ES, here too the prefactor $F\left(t ; t^{\prime}\right)$ of the Gaussian $\rho_{0}\left(t ; t^{\prime}\right)$ cannot be factorized into $(N-n)$ two HO density matrices. However, as discussed in Appendix C 2, if the vector $t^{T}$ is outside the maximum $t_{\max }^{T}$, given by Eq. (C19) corresponding to the $3 \sigma$ limits, the argument of $\rho_{0}\left(t ; t^{\prime}\right)$ is negligible. Therefore, if the conditions (C18) as well as the conditions

$$
\tilde{\boldsymbol{\epsilon}}_{1} \equiv t_{\max }^{T} \Lambda_{\beta^{\prime}} t_{\max } \ll 1, \quad \tilde{\epsilon}_{2} \equiv t_{\max }^{T} \Lambda_{\gamma^{\prime}} t_{\max } \ll 1
$$

are satisfied, then we can approximate the prefactor $F\left(t ; t^{\prime}\right)$ as

$$
F\left(t ; t^{\prime}\right) \approx \exp \left[\tilde{\kappa}_{1} w\left(t ; t^{\prime}\right)+\tilde{\kappa}_{2} v\left(t ; t^{\prime}\right)\right],
$$

where we have kept terms up to quadratic order in $t, t^{\prime}$. (Note that $v\left(t ; t^{\prime}\right)$ is only linear in $t, t^{\prime}$ whereas $w\left(t ; t^{\prime}\right)$ is quadratic in $t, t^{\prime}$.)

Using Eq. (C4) for $\rho_{0}\left(t ; t^{\prime}\right)$ we can now write the (approximated) MS density matrix as

$$
\rho\left(t ; t^{\prime}\right)=\tilde{\kappa} \sqrt{\frac{|\bar{\Omega}|}{\pi^{N-n}|A|}} \exp \left[z\left(t ; t^{\prime}\right)+\tilde{\kappa}_{2} v\left(t ; t^{\prime}\right)\right]
$$

where

$$
z\left(t ; t^{\prime}\right)=-\frac{t^{T} \gamma^{\prime} t+t^{\prime T} \gamma^{\prime} t^{\prime}}{2}+t^{T} \beta^{\prime} t^{\prime}
$$

and

$$
\begin{aligned}
& \beta^{\prime}=\beta+\tilde{\kappa}_{1} \Lambda_{\beta^{\prime}}=\beta+\tilde{\kappa}_{1} \Lambda_{\beta}-2 \tilde{\kappa}_{0} \tilde{\kappa}_{1}\left(\Lambda_{\beta}-\frac{\Lambda_{C}}{\kappa}\right), \\
& \gamma^{\prime}=\gamma+\tilde{\kappa}_{1} \Lambda_{\gamma^{\prime}}=\gamma+\tilde{\kappa}_{1} \Lambda_{\gamma}+2 \tilde{\kappa}_{0} \tilde{\kappa}_{1}\left(\Lambda_{\beta}-\frac{\Lambda_{C}}{\kappa}\right)
\end{aligned}
$$

are $(N-n) \times(N-n)$ matrices. $\beta^{\prime}$ is symmetric while $\gamma^{\prime}$ is not necessarily symmetric.

Let us make the following transformations on the set of $(N-n) \quad$ variables $t \equiv\left\{x_{n+1}, \cdots, x_{N}\right\} \quad$ and $\quad t^{\prime} \equiv$ $\left\{x_{n+1}^{\prime}, \cdots, x_{N}^{\prime}\right\}$ :

$$
t \rightarrow t+s, \quad t^{\prime} \rightarrow t^{\prime}+s
$$

where $s \equiv\left\{s_{1}, \cdots, s_{N-n}\right\}$ is a set of $(N-n)$ constant values. The density matrix (29) reduces to

$$
\rho\left(t ; t^{\prime}\right)=\mathcal{N} \exp \left[-\frac{t^{T} \gamma^{\prime} t+t^{\prime T} \gamma^{\prime} t^{\prime}}{2}+t^{T} \beta^{\prime} t^{\prime}\right]
$$

where the normalization constant $\mathcal{N}$ is given by

$$
\mathcal{N}=\tilde{\kappa} \sqrt{\frac{|\Omega|}{\pi^{N-n}|A|}} \exp \left[-s^{T}\left(\beta^{\prime}-\gamma^{\prime}\right)^{T} s\right] .
$$

The $(N-n)$-dimensional constant column vector $s$ is determined from the equation

$$
s^{T}\left(\beta^{\prime}-\frac{\gamma^{\prime}+\gamma^{\prime T}}{2}\right)=-\tilde{\kappa}_{2}\left(y_{B}-B^{T} A^{-1} y_{A}\right) .
$$

It is easy to check that for either $c_{0}=0$ or $c_{1}=0$, the constant $k_{2}$ vanishes, whence $s=0$, and the density matrix (33) reduces either to that of pure GS [7] (for $c_{0}=1, c_{1}=$ 0 whence $\beta^{\prime}=\beta, \gamma^{\prime}=\gamma$ ) or that of ES [14] (for $c_{0}=0$, $c_{1}=1$, whence $\beta^{\prime}=\beta+\Lambda_{\beta}, \gamma^{\prime}=\gamma+\Lambda_{\gamma}$ ). In general, when both $c_{0}$ and $c_{1}$ are nonvanishing, then under the shifts $\beta \rightarrow \beta^{\prime}, \gamma \rightarrow \gamma^{\prime}$ [where $\beta^{\prime}$ and $\gamma^{\prime}$ are given by Eq. (31)] the MS density matrix (33) is of the same form as the GS density matrix (C4), up to a normalization factor given above. Such a normalization constant does not affect the entropy computation. Therefore we can use the same steps as for GS [Eqs. (C5)-(C9), with the replacements $\beta \rightarrow \beta^{\prime}$, $\left.\gamma \rightarrow \gamma^{\prime}\right]$ to calculate the total MS entropy.

The rest of the analysis in this section is similar to that of Ref. [14]. We compute the entanglement entropy numerically (using MATLAB) in each of the cases:

(i) GS $\left(c_{0}=1, c_{1}=0\right)$

(ii) $\mathrm{ES}\left(c_{0}=0, c_{1}=1\right)$ 
In $S$ vs In (R/a) plots for GS, ES \& MS(Eq/Hi)
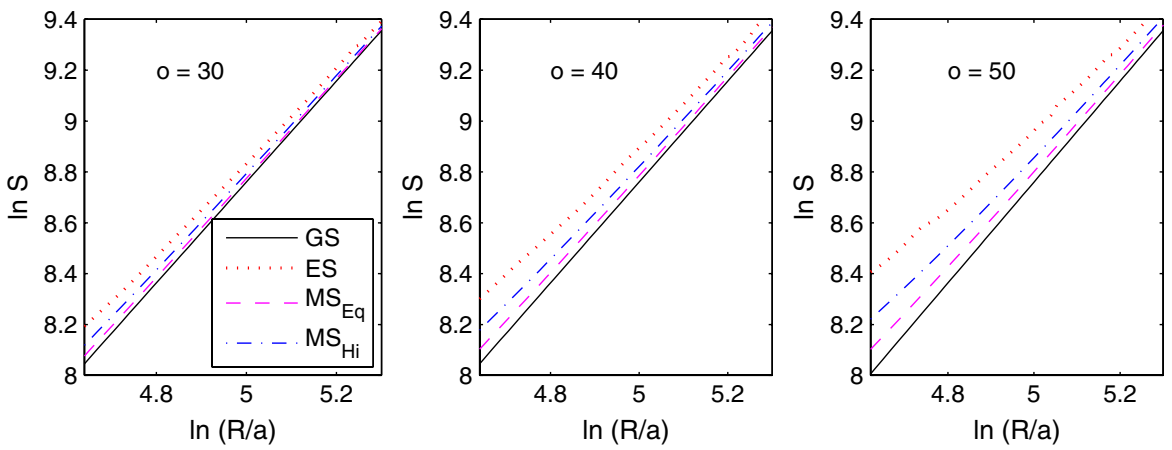

FIG. 1 (color online). Plots of logarithm of GS, ES, and MS (Eq/Hi) entropies versus $\ln (R / a)$, where $R=a(n+1 / 2)$ radius of the hypothetical sphere (horizon), for $N=300, n=100-200$ and $o=30,40,50$ (in the ES and MS cases). The numerical precision is $0.01 \%$.

(iii) an equal mixing $\left(\mathrm{MS}_{\mathrm{Eq}}\right)$ of ES with $\mathrm{GS}\left(c_{0}=c_{1}=\right.$ $1 / \sqrt{2})$, and

(iv) a high mixing $\left(\mathrm{MS}_{H i}\right)$ of ES with $\mathrm{GS}\left(c_{0}=1 / 2\right.$, $\left.c_{1}=\sqrt{3} / 2\right)$.

The computations have been done with a precision ${ }^{4}$ of $0.0 \%$ for the set of values: $N=300, n=100-200$ and $o=$ $30,40,50 o$ being the last nonzero columns of the vector $\alpha^{T}$. The conditions (C18) as well as (27) are satisfied for these values of the parameters.

The expectation value of energy, $\mathcal{E}$, for MS can be expressed as

$$
\mathcal{E}=\mathcal{E}_{0}+\frac{c_{1}^{2}}{o} \sum_{i=N-o+1}^{N} k_{D i}^{1 / 2}
$$

where $\mathcal{E}_{0}=\frac{1}{2} \sum_{i=1}^{N} k_{D i}^{1 / 2}$ is the (zero-point) GS energy. Now the excess of energy over the zero-point energy is given by the second term in the above Eq. (36). As the value of $c_{1}$ is between 0 and 1 and since the $k_{D i}$ 's are in ascending order $\left(k_{D i}>k_{D j}\right.$ for $\left.i>j\right)$, the fractional change in energy $(\mathcal{E}-$ $\left.\mathcal{E}_{0}\right) / \mathcal{E}$ is at most about $\sim 5 \%$ (corresponding to the extreme situation $c_{1}=1$, i.e., ES), for $N=300$ and $o \sim 50$. Moreover, since there are $o$ number of terms in the sum in the second term of Eq. (36), the excitation energy $(\mathcal{E}-$ $\mathcal{E}_{0}$ ) is of the order unity (in units of $1 / a$, where $a$ is the lattice spacing). Therefore if $a$ is chosen to be of the order of the Planck length, then the above energy is of the order of the Planck energy. The mass of a semiclassical $\mathrm{BH}$, on the other hand, is much larger than the Planck mass. Hence, one may safely neglect the backreaction of the scalar field on the background geometry.

In Fig. 1, we have plotted the logarithm of the entropy $S$ versus $\ln (R / a)=\ln (n+1 / 2)$, for different values of the excitation $(o=30,40,50)$, for GS, ES, and MS (Eq/Hi).

\footnotetext{
${ }^{4}$ The computations here are 1 order of magnitude more accurate than those in Ref. [14]
}

For GS, the plot is very nearly the same as the numerical straight line fit obtained in Ref. [7], $S=0.3(R / a)^{2}$ with $N=60$ lattice points. For the MS (Eq/Hi) cases, as well as for ES, the plots are nearly linear for different values of the excitations $o=30,40,50$ and appear to coincide with the plot for GS for large areas $\left(\mathcal{A}=4 \pi R^{2} \gg a^{2}\right)$. Numerical straight line fittings of the logarithm of the ES entropy, $S_{\mathrm{ES}}$, with $\ln (R / a)$ shown in [14] revealed that for smaller areas $S_{\mathrm{ES}} \sim A^{\mu}$, where $\mu$ is always $<1$ and decreases as the number of excitation $o$ increases. To have a closer look at the behavior of MS entropy, $S_{\mathrm{MS}}$, (for both equal and high mixings) and the ES entropy $S_{\mathrm{ES}}$ with respect to the GS entropy, $S_{\mathrm{GS}}$, we have plotted in Fig. 2 the ratios $S_{\mathrm{MS}} / S_{\mathrm{GS}}$, $S_{\mathrm{ES}} / S_{\mathrm{GS}}$ and the inverse ratios $S_{\mathrm{GS}} / S_{\mathrm{MS}}, S_{\mathrm{GS}} / S_{\mathrm{ES}}$, versus the area $\mathcal{A}$. For the range of excitations $(o=30,50)$, all the ratios tend to unity as the area increases. Thus the general criterion of "asymptotic equivalence" [17] is fulfilled, i.e.,

$$
\lim _{\mathcal{A} \rightarrow \infty} \frac{S_{\mathrm{XS}}(\mathcal{A})}{S_{\mathrm{GS}}(\mathcal{A})}=1, \quad \lim _{\mathcal{A} \rightarrow \infty} \frac{S_{\mathrm{GS}}(\mathcal{A})}{S_{\mathrm{XS}}(\mathcal{A})}=1
$$

where XS $\equiv \mathrm{MS}$ (Eq or Hi) or ES. In other words, the MS $(\mathrm{Eq} / \mathrm{Hi})$ and the ES entropies coincide asymptotically with the GS entropy. However, as is evident from Fig. 2, the $\mathrm{MS}(\mathrm{Eq})$ entropy is closer to the GS entropy for large $\mathcal{A}$, than the MS(Hi) entropy and the ES entropy, the latter being the farthest. This implies that the asymptotic behavior is strongly influenced by the relative weight $c_{1}$ of the mixing of ES with GS - the smaller the value of $c_{1}$ the sharper is the asymptote.

In order to make things more transparent we have plotted in Fig. 3 the best fit ratios of the MS entropies (for equal and high mixings, with $o=30,40,50$ ) to the GS entropy using a simple formula:

$$
\frac{S_{\mathrm{MS}}}{S_{\mathrm{GS}}}=\sigma_{0}+\sigma\left(\frac{\mathcal{A}}{a^{2}}\right)^{-\nu} .
$$

The fitting parameters $\sigma_{0}, \sigma$, and $\nu$ are shown in Table I. 

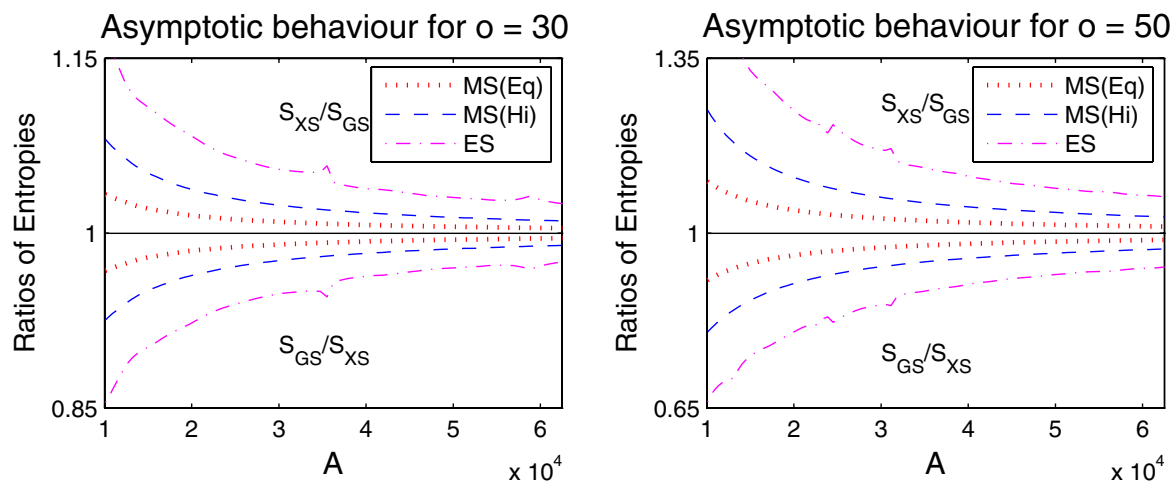

FIG. 2 (color online). Plots of ratios of GS and MS (Eq/Hi) or ES entropies and their reciprocals versus the area $\mathcal{A}$ (in units of $a^{2}, a$ being the lattice spacing) for $o=30,50$. The plots show the asymptotic nature of the MS and ES entropies with respect to the GS entropy. The curves on the upper half (above 1) show the variation of $S_{\mathrm{XS}} / S_{\mathrm{GS}}$ with $\mathcal{A}$, where XS stands for MS(Eq/Hi) or ES, while the lower curves show the variation of $S_{\mathrm{GS}} / S_{\mathrm{XS}}$ with $\mathcal{A}$.
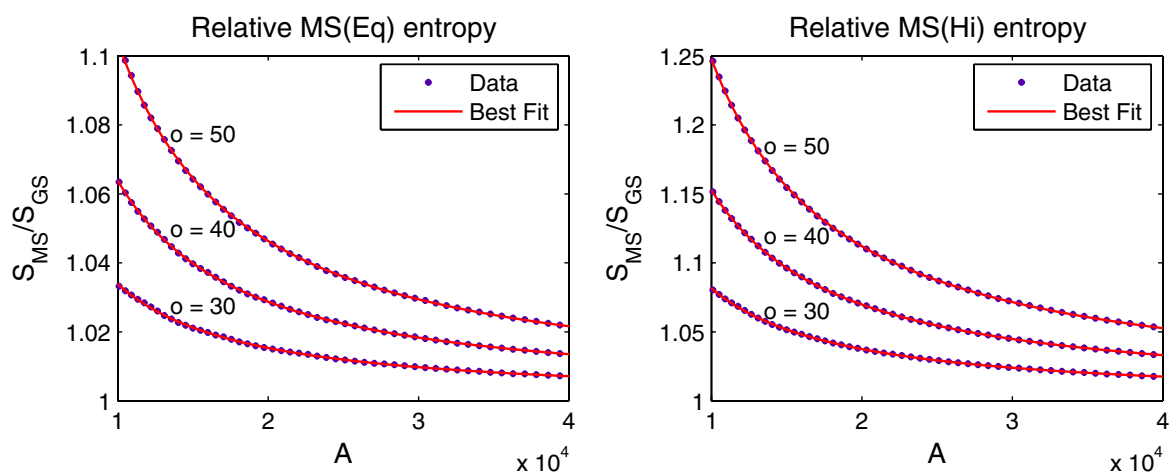

FIG. 3 (color online). Best fit plots (solid lines) of the relative mixed state entropies $\left(S_{\mathrm{MS}} / S_{\mathrm{GS}}\right)$ for equal and high mixings versus the area $\mathcal{A}$ (in units of $a^{2}$ ), for $o=30,40,50$. The corresponding data are shown by asterisks.

The parameter $\sigma_{0}$ is very close to unity for all values of $o=30,40,50$, for both $\mathrm{MS}(\mathrm{Eq})$ and $\mathrm{MS}(\mathrm{Hi})$ cases. However, there is a slight increase in $\sigma_{0}$ as $o$ increases or for greater relative weight $c_{1}$ of mixing of ES with GS for a particular $o$ [i.e., $\sigma_{0}$ is greater in the $\mathrm{MS}(\mathrm{Hi})$ case than in the $\mathrm{MS}(\mathrm{Eq})$ case for fixed $o$ ]. Neglecting this variation in $\sigma_{0}$ and noting that the GS entropy can be written as $S_{\mathrm{GS}}=$ $n_{0}\left(\mathcal{A} / a^{2}\right)$, where $n_{0}$ is a constant, we can approximately express

$$
S_{\mathrm{MS}}=S_{\mathrm{GS}}+\tilde{\sigma}\left(\frac{\mathcal{A}}{a^{2}}\right)^{1-\nu},
$$

where $\tilde{\sigma}=n_{0} \sigma$. As the value of the exponent $(1-\nu)$ lies between 0 and -1 for both equal and high mixings (see Table I) the second term in the above Eq. (39) may be regarded as a power-law correction to the $\mathrm{AL}$, resulting from entanglement, when the wave function of the field is chosen to be a superposition of GS and ES. It is important to note that the correction term falls off rapidly with $\mathcal{A}$ (due to the negative exponent) and in the semiclassical limit $(\mathcal{A} \gg 1)$ the $\mathrm{AL}$ is hence recovered. This lends further credence to entanglement as a possible source of black-hole entropy. The correction term is more significant for higher excitations $o$ or greater ES-GS mixing proportion $c_{1}$. This is evident from Table I, which shows that the parameter $\sigma$ (and hence $\tilde{\sigma}$ ) increases and the parameter $\nu$

TABLE I. Values of the parameters of the fit $S_{\mathrm{MS}} / S_{\mathrm{GS}}=\sigma_{0}+\sigma\left(\mathcal{A} / a^{2}\right)^{-\nu}$ for both MS(Eq) and $\mathrm{MS}(\mathrm{Hi})$ cases with the amounts of excitation $o=30,40,50$.

\begin{tabular}{lcccccc}
\hline \hline Fitting Parameters & \multicolumn{3}{c}{${\text { For } \mathrm{MS}_{\mathrm{Eq}}}$} & \multicolumn{3}{c}{ For $\mathrm{MS}_{\mathrm{Hi}}$} \\
\hline & $o=30$ & $o=40$ & $o=50$ & $o=30$ & $o=40$ & $o=50$ \\
$\sigma_{0}$ & 1.001 & 1.002 & 1.003 & 1.001 & 1.004 & 1.006 \\
$\sigma$ & 1738 & 4288 & 8039 & 2956 & 7652 & 14120 \\
$\nu$ & 1.180 & 1.210 & 1.225 & 1.141 & 1.178 & 1.192 \\
\hline \hline
\end{tabular}


$(2 I+1) S_{\text {I }}$ vs I for GS

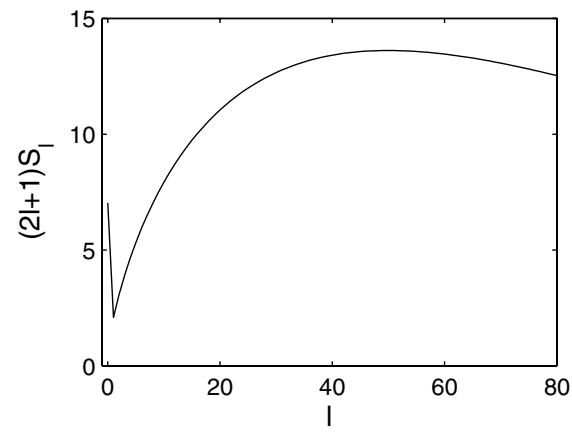

$(2 \mathrm{l}+1) \mathrm{S}_{\mathrm{I}}$ vs I for $\mathrm{MS}_{\mathrm{Eq}}$

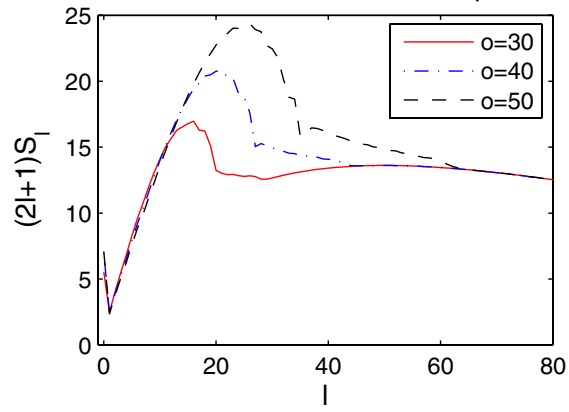

$(2 \mathrm{I}+1) \mathrm{S}_{\text {, vs I for } \mathrm{ES}}$

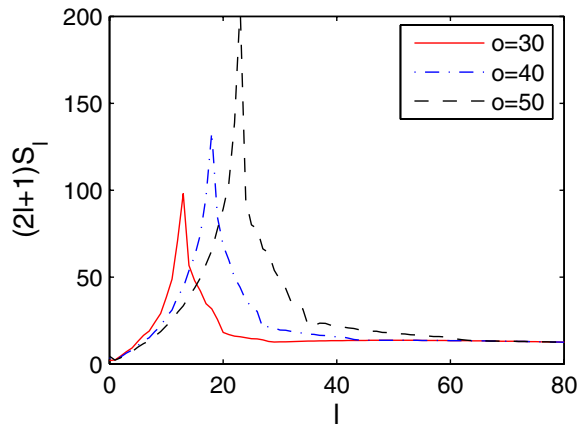

$(2 \mathrm{l}+1) \mathrm{S}_{\mathrm{l}}$ vs I for $\mathrm{MS}_{\mathrm{Hi}}$

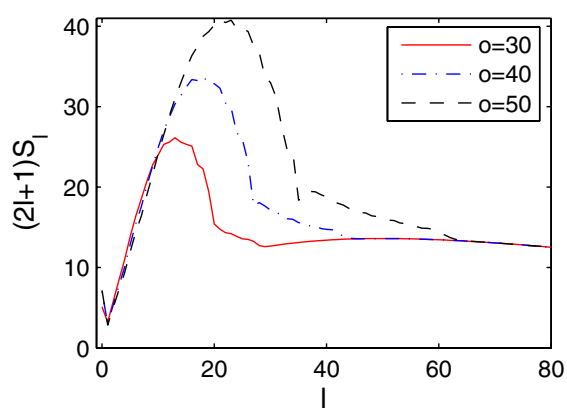

FIG. 4 (color online). Plots of the distribution of entropy per partial wave $\left[(21+1) S_{l}\right]$ in the cases of GS, ES, and MS (Eq/Hi), for $N=300, n=100$, and $o=30,40,50$.

(and hence the negative exponent $|1-\nu|$ ) decreases with the increase in $o$ (fixed $c_{1}$ ) or the increase in $c_{1}$ (fixed $o$ ).

Figure 4 shows the variation of $(2 l+1) S_{l}$ with $l$, in the cases of GS, ES, and MS (Eq/Hi) for a fixed $n(=100)$ and a set of increasing values of $o$. For the GS, there is a peak at $l=0$ ( $s$-wave), followed by another one at $l \approx 40$ due to the degeneracy factor $(2 l+1)$. The first peak shifts to a value $l>0$ for the ES, and the shift is greater as $o$ is increased. There is, however, no second peak in this case, although there seems to be an increase towards higher values of $l$. Thus, higher partial waves are seen to get excited with greater excitations. In each of the MS cases, there is a trace of the first peak at $l=0$ as for GS, however the amplitude of that peak is very small compared to the second peak which appears between $l \sim 10-30$ depending on the value of the excitations $o$. As in the case of ES, the second peaks for MS (Eq/Hi) are higher and far away from $l=0$ for increasing values of $o$. However, relative to the ES case, there is a broadening of the half-width of the peaks for MS, though not as broad as that of the second peak for GS. Thus, as expected, the $(2 l+1) S_{l}$ vs $l$ curves for MS show features that are intermediate between those for GS and ES.

\section{LOCATION OF THE DEGREES OF FREEDOM}

Let us now examine closely the expression for the interaction matrix $K_{i j}$, Eq. (9), for the system of $N$ HOs. The last two terms, which signify the nearest-neighbor
(NN) interaction between the oscillators, are solely responsible for the entanglement entropy of black holes, i.e., $S_{\mathrm{BH}}=0$ if these two terms are set to zero. Let us, however, consider the situation where the $\mathrm{NN}$ interactions, and hence the off-diagonal elements of $K_{i j}$, are set to zero (by hand) everywhere except in a "window," such that the indices $i, j$ run from $q-s$ to $q+s$, where $s \leq q$. Thus the interaction region is restricted to a width of $d=2 s+1$ radial lattice points. Now, choosing the position of the center of the window $q$ to vary between 0 and a value $q_{\text {max }}>n$, we allow the window to move rigidly across from the origin to a point outside the horizon. Figure 5 shows the variation of the percentage contribution of the entropy for a fixed window size of 5 lattice points $(d=5$, $s=2$ ), i.e.,

$$
p c(q)=\frac{S(q, d=5)}{S_{\mathrm{tot}}} \times 100
$$

as a function of $q$ for fixed values $N=300, n=100$ in each of the cases GS, ES, and MS (Eq/Hi) with $o=30,50$. Here $S_{\text {tot }}$ is the total entropy with all the $\mathrm{NN}$ interactions present, i.e., $i, j$ running from 0 to $N$.

In all the cases of GS, ES, and $\mathrm{MS}(\mathrm{Eq} / \mathrm{Hi})$ the first observation is that $p c(q)=0$ when $q$ is far away from $n$. There is no contribution to the total entanglement entropy if the interaction window does not include the horizon. For values of $q$ very close to $n$ there are significant contributions to $S_{\text {tot }}$ and in the case of GS, $p c(q)$ peaks exactly at 

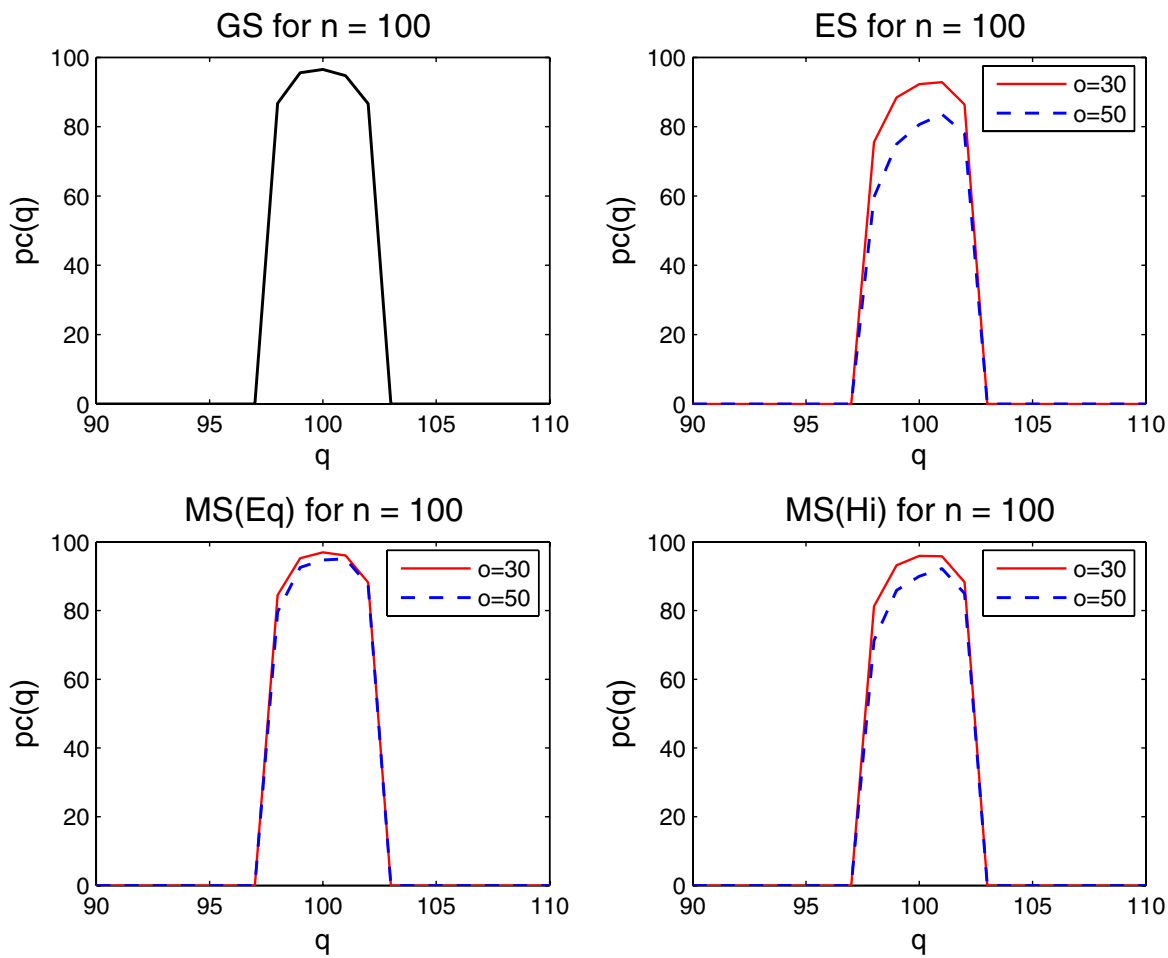

FIG. 5 (color online). Plots of the percentage contribution $p c(q)$ to the total entropy as a function of window position $q$, for a window size $d=5$ and fixed $N=300, n=100$, in each of cases of GS, ES, and MS (Eq/Hi). For ES and MS (Eq/Hi) the solid curve is for $o=30$ whereas the broken curve is for $o=50$.

$q=n$. For ES and MS, however, the peaks tend to shift towards a value $q>n$, their exact positions depending on the amount of excitation $o$. Moreover, the amplitudes of the peaks gradually diminish as the value of $o$ and/or the mixing weight $c_{1}$ increases. Thus, we infer that:

(i) The contribution to the total entropy is more from the DOF that are in the vicinity (inside or outside) of the horizon, rather than far from it.

(ii) The contributions, however small, from the DOF far away from the horizon are more for $\mathrm{MS}(\mathrm{Eq} / \mathrm{Hi})$ and ES, compared to the GS. In other words, the contributions from the far-away DOF increases with increase in the number of excitations and amount of mixing of ES with GS.

Further investigations have been carried out recently in Ref. [16] to check the effects of the far-away DOF on the total entropy, by keeping fixed the center of the window at the horizon, i.e., $q=n$, while increasing the window width $d$ from 0 to $n$. It is found that for GS about $85 \%$ of the total entropy is obtained within a width of just one lattice spacing, and within a width of $d=3$ almost the entire GS entropy is recovered. Thus most of the GS entropy comes from the DOF very close to the horizon and a small part (about 15\%) has its origin deeper inside. For ES, however, the corresponding figures are about $60 \%(d=$ 5 ), and the total ES entropy is recovered when $d$ is as much as $15-20$, depending on the number of excitations $o=$ 30-50. Thus the far-away DOF contribute more to the entropy for the ES. This, in turn, may be looked upon as follows: the larger the deviation from the area law, the larger is the contribution to the total entropy from the DOF that are far away from the horizon. The situation is intermediate for the MS (which itself interpolates between the GS and ES): This is evident from Fig. 5 (and also from Fig. 1) where unlike the curves for ES, those for the MS cases do not show much deviations from the curve for GS, even for high excitations $o$.

\section{ENTANGLEMENT ENTROPY OF MASSIVE SCALAR FIELD}

As shown in Appendix A, the equation of motion for metric perturbations in a general space-time with a cosmological constant $|\Lambda|$ coincides with that of a test massive scalar field propagating in the background metric. In all our earlier analysis, we had set, for simplicity, $|\Lambda|=0$. In this section, we obtain the entanglement entropy for the massive scalar field.

The action for the massive scalar field (mass $m$ ) propagating in the background space-time $g_{\mu \nu}$ is

$$
S=-\frac{1}{2} \int d^{4} x \sqrt{-g}\left[g^{\mu \nu} \partial_{\mu} \varphi \partial_{\nu} \varphi+m^{2} \varphi^{2}\right]
$$

Repeating the steps described in Appendix B for the massive scalar will lead to massive, free field Hamiltonian (B15). Discretizing the resulting Hamiltonian, as described 

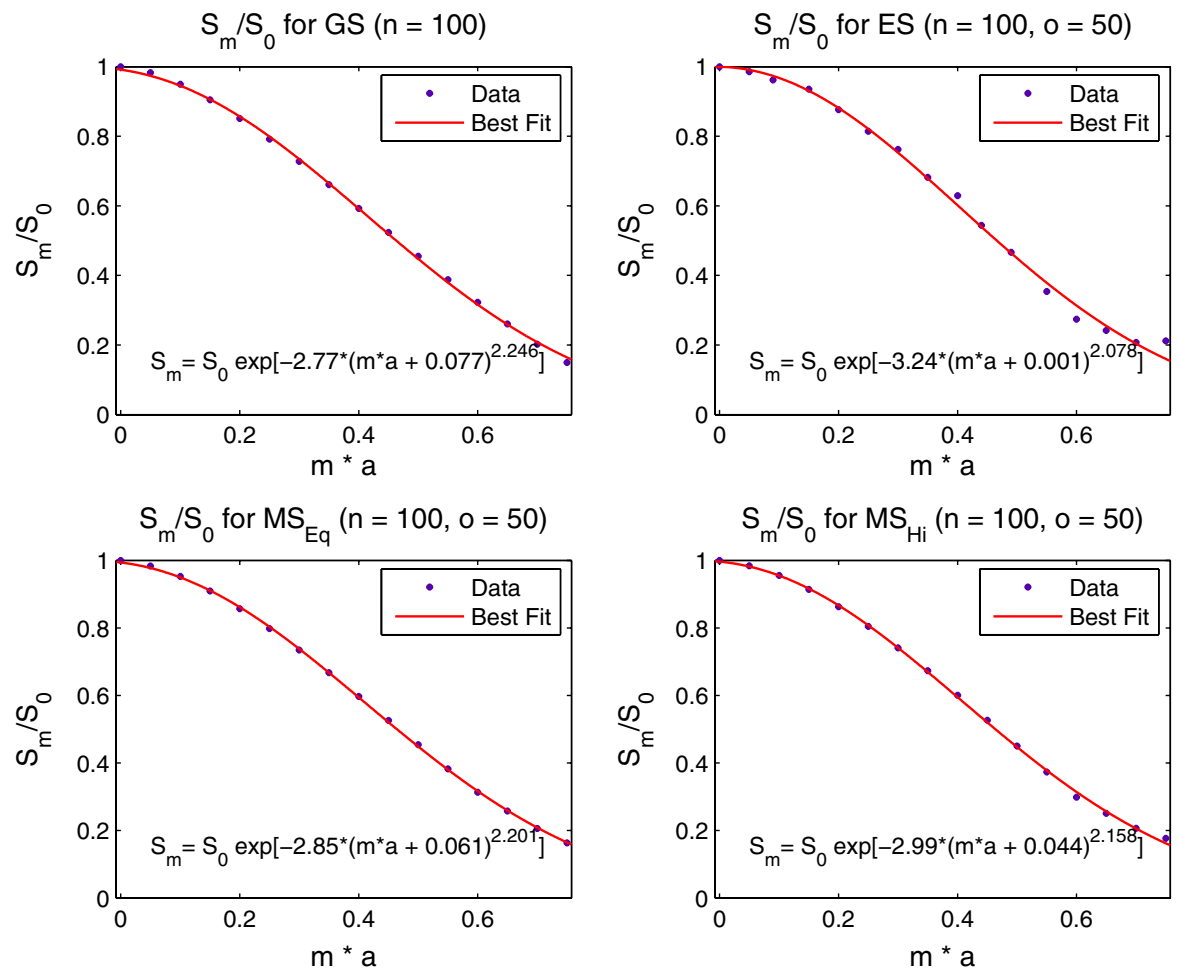

FIG. 6 (color online). Best fit plots of the relative variation of the total entropy $S_{m}$ for a massive scalar field (in units of the total entropy $S_{0}$ corresponding to a massless scalar field) with the mass $m$ times the lattice spacing $a$, for fixed $n=100, o=30$, in each of cases of GS, ES, and MS (Eq/Hi). The corresponding data are shown by asterisks. The fits show an exponential damping of the ratio $S_{m} / S_{0}$ with mass.

in Sec. II will lead to the $N$ coupled HO Hamiltonian, with the interaction matrix $K_{i j}^{(m)}$ given by

$$
K_{i j}^{(m)}=K_{i j}+(m a)^{2}
$$

where $K_{i j}$ is the interaction matrix, given by Eq. (9), for a massless scalar field. Following the steps discussed in Secs. II and III, we can compute the entanglement entropy for the massive field $\left(S_{m}\right)$. In Fig. 6, we have plotted $S_{m} / S_{0}$ (where $S_{0}$ is the entropy for the massless scalar) for the GS, $\mathrm{ES}$, and $\mathrm{MS}(\mathrm{Eq} / \mathrm{Hi})$ for $N=300, n=100, o=50$. The Gaussian fits indicate an exponential fall-off of $S_{m}$ with respect to $S_{0}$ as the mass increases:

$$
S_{m}=S_{0} \exp \left[-\alpha_{1}\left(m a+\alpha_{2}\right)^{\lambda}\right]
$$

where $\alpha_{1}, \alpha_{2}$, and $\lambda$ are the fitting parameters. Depending on the state (GS, ES, or MS), the parameter $\alpha_{1}$ varies between 2.77 and 3.24, $\alpha_{2}$ is between 0.077 and 0.001 , and the power $\lambda$ is close to 2 . Thus approximately $S_{m} / S_{0}$ scales as $e^{-m^{2} a^{2}}$. There is, however, a small variation in the power $\lambda$ for the different cases. The exponential damping is strongest for GS, and gradually slows down as more and more ES oscillators are mixed with GS, the damping is slowest for the ES case.

Although $S_{m}$ scales as $S_{0}$ times a mass-dependent exponential term, the fitting parameters $\alpha_{1}, \alpha_{2}$, and $\lambda$ change very little for the different cases GS, MS(Eq/Hi), and ES, even for a fairly high amount of excitation $o=50$. As such, for a fixed mass $m$, the variations $\ln S$ vs $\ln (R / a)$ for all the cases remain almost the same as those for massless scalar field, cf. Fig. 1. The analysis and inferences of the previous sections for the massless scalar go through for the massive scalar field, resulting in correction terms obtained before.

\section{CONCLUSIONS}

In this work, we have obtained power-law corrections to entanglement entropy, which may be relevant for the entropy of BH. Indeed, as shown in Appendices A and B, certain modes of gravitational perturbations in black-hole space-times behave as minimally coupled scalar fields. Also for computation of time-independent quantities done at a fixed value of Lemaître time, it suffices to consider an effective flat space Hamiltonian. Extending the analysis of the earlier work [14], we have shown that for small black-hole areas the area law is violated not only when the oscillator modes that represent the scalar DOF are in ES, but also when they are in a linear superposition of GS and ES. We found that the corrections to the AL become increasingly significant as the proportion of ES in the superposed state increases. Conversely, for large horizon areas, these corrections are relatively small and the $\mathrm{AL}$ is recovered. 
It is interesting to compare and contrast the power-law corrections obtained here to those derived in the case of higher-derivative gravity [15]. The power-law corrections to the Bekenstein-Hawking entropy derived in both-entanglement and higher-derivative gravity-these approaches have the same features. For instance, it was shown that the entropy of a 5-dimensional BoulwareDeser black hole [18] is given by

$$
S=\frac{A}{4}\left[1+\frac{c}{A^{2 / 3}}\right], \quad c=\text { constant }
$$

As in Eq. (39) the above entropy is proportional to area for large horizon radius, however it strongly deviates in the small horizon limit. It is important to note that the corrections to the black-hole entropy are generic and valid even for black holes in general relativity without any higher curvature terms. ${ }^{5}$ It is interesting to investigate the relation between the entanglement entropy with that of the Noether charge approach [19].

The location of the DOF that give rise to the entanglement entropy has important implications as far as the corrections to the AL is concerned. It is found that for GS, ES, and MS, the contributions to the total entropy from the DOF that are nearest to the horizon are maximum. However, there are small contributions from DOF that are far away from the horizon, which also need to be taken into account in order for the AL to emerge. These contributions are least in the case of GS and gradually increase as the proportion of mixing of ES with GS and/or the amount of excitation increases. Correspondingly, there are increasing deviations from the AL. Thus one is led to conclude that the AL is intimately linked with near horizon DOF.

We have also shown that the mass of the scalar field does not have much influence on the corrections to the AL. The total entropy for the massive field scales as that of the massless field times a mass-dependent exponentially damping term that varies very slowly with the mixing proportion and the amount of excitation which are key to producing the AL corrections.

Open problems in the context of entanglement entropy include: (i) The proportionality constant in the relation $S=$ $0.3(R / a)^{2}$ for GS obtained in Ref. [7] differs from the $1 / 4$ in the Bekenstein-Hawking relation [Eq. (2)]. This discrepancy persists for MS and ES. A probable reason behind this mismatch is the dependence of the prefactor on the type of the discretization scheme. For example, another discretization scheme, resulting in the NN interactions between four or more immediate neighbors, would result in a different prefactor. Is it then at all possible to obtain the Bekenstein-Hawking value? (ii) Can a temperature emerge in the entanglement entropy scenario, and if so, then along

\footnotetext{
${ }^{5}$ In this context, it should be mentioned that it is not possible to check for logarithmic corrections to the entropy in our analysis, as the numerical error we obtain is much larger than $\ln (n+1 / 2)$.
}

with the current entropy, will it be consistent with the first law of BH thermodynamics? (iii) Are the second and third laws of thermodynamics valid for this entropy? (iv) Can the entanglement of scalar fields help us to understand the evolution or dynamics of BHs and the information loss problem? We hope to report on these in the future.

\section{ACKNOWLEDGMENTS}

The works of S.D. and S. Sur are supported by the Natural Sciences and Engineering Research Council of Canada.

Note added. - Recently, in Ref. [20], the authors have calculated the subleading power-law corrections to the Bekenstein-Hawking entropy using the canonical ensemble (aka brick wall) approach [8]. The results reported there agree with the numerical results derived in this work.

\section{APPENDIX A: WHY CONSIDER SCALAR FIELDS?}

In this appendix, we briefly discuss the motivation for obtaining the entanglement entropy of a scalar field. First, we obtain the equation of motion of the metric perturbations for a general space-time and then, as a special case, discuss the equation of motion of the perturbations in asymptotically flat spherically symmetric space-times.

Consider the Einstein-Hilbert action with a positive cosmological constant $(|\Lambda|)$ :

$$
S_{\mathrm{EH}}(\bar{g})=M_{\mathrm{Pl}}^{2} \int d^{4} x \sqrt{-\bar{g}}[\bar{R}-2|\Lambda|] .
$$

Letting $\bar{g}_{\mu \nu}=g_{\mu \nu}+h_{\mu \nu}$ and expanding the action keeping only the parts quadratic in $h_{\mu \nu}$, we get [21]

$$
\begin{aligned}
S_{\mathrm{EH}}(g, h)= & -M_{\mathrm{Pl}}^{2} \int d^{4} x \sqrt{|g|}\left[2 \gamma^{\alpha}{ }_{\mu \nu} \gamma_{\alpha}^{\mu \nu}{ }_{\alpha}+\frac{1}{4} \nabla_{\mu} \tilde{h} \nabla^{\mu} \tilde{h}\right. \\
& \left.+\frac{|\Lambda|}{2} h_{\mu \nu} \tilde{h}^{\mu \nu}\right]
\end{aligned}
$$

where

$$
\begin{gathered}
\tilde{h}_{\mu \nu} \equiv h_{\mu \nu}-\frac{1}{2} g_{\mu \nu} h_{\alpha}^{\alpha}, \quad \tilde{h} \equiv \tilde{h}_{\mu}^{\mu}, \\
\gamma_{\mu \nu}^{\alpha} \equiv \frac{1}{2}\left(\nabla_{\mu} \tilde{h}_{\nu}^{\alpha}+\nabla_{\nu} \tilde{h}_{\mu}^{\alpha}-\nabla^{\alpha} \tilde{h}_{\mu \nu}\right) .
\end{gathered}
$$

The above action is invariant under the infinitesimal gauge transformation $h_{\mu \nu} \rightarrow h_{\mu \nu}+\nabla_{(\mu} \xi_{\nu)}$ when the background metric $g_{\mu \nu}$ satisfies the vacuum Einstein's equation with $|\Lambda|$. We can remove the gauge arbitrariness by imposing the harmonic gauge condition $\partial_{\mu} \tilde{h}^{\mu \nu}=0$ [21].

Assuming $h_{\mu \nu}$ to be small, we can keep only the first derivatives of $h_{\mu \nu}$. With these two conditions, the action (A2) reduces to (for more details see Ref. [22], pp. 330332) 


$$
\begin{aligned}
S_{\mathrm{EH}}(g, h)= & -\frac{M_{\mathrm{Pl}}^{2}}{2} \int d^{4} x \sqrt{|g|}\left[\nabla_{\alpha} h_{\mu \nu} \nabla^{\alpha} h^{\mu \nu}\right. \\
& \left.+|\Lambda| h_{\mu \nu} h^{\mu \nu}\right] .
\end{aligned}
$$

The above action corresponds to the massive spin- $2 h_{\mu \nu}$ field propagating in the background metric $g_{\mu \nu}$ where the cosmological constant appears as mass term. In the weak field limit — when the gravitational field is weak like in the case of regions close to the black-hole horizons $-h^{\mu \nu}$ can be approximated as a plane-wave perturbation with a particular frequency, i. e., $h_{\mu \nu}=M_{\mathrm{Pl}}^{-1} \epsilon_{\mu \nu} \varphi\left(x^{\mu}\right)$ (where $\epsilon_{\mu \nu}$ is the constant polarization tensor), the above action can be written as

$$
S_{\mathrm{EH}}(g, h)=-\frac{1}{2} \int d^{4} x \sqrt{|g|}\left[\partial_{\alpha} \varphi \partial^{\alpha} \varphi+|\Lambda| \varphi^{2}\right] .
$$

which is the action for the massive scalar field propagating in the background metric $g_{\mu \nu}$.

Moreover, for four-dimensional spherically symmetric space-times, the metric perturbations are of two kindsaxial and polar [23-25]. The equations of motion of both these perturbations are scalar in nature and are related to each other by a unitary transformation [23]. The equation of motion of the axial perturbations is nothing but the equation of motion of a test, massless scalar field propagating in the black-hole background:

$$
\square \varphi \equiv \frac{1}{\sqrt{-g}} \partial_{\mu}\left(\sqrt{-g} g^{\mu \nu} \partial_{\nu} \varphi\right)=0 .
$$

Hence, by computing the entanglement entropy of the scalar fields, we obtain the entropy of a class of metric perturbations of the background space-time. Of course, a generic perturbation being a superposition of plane-wave modes, and entanglement entropy being a nonlinear function of the wave function, we do not claim that such a computation would account for the entropy of all perturbations. Nevertheless, it is expected to shed important light on the role of entanglement in the AL.

In most parts of this work, we calculate the entropy of the massless scalar field (i.e., setting $|\Lambda|=0$ ). In Sec. V, we obtain the entanglement entropy of the massive field, corresponding to $|\Lambda| \neq 0$.

\section{APPENDIX B: HAMILTONIAN OF SCALAR FIELDS IN BLACK-HOLE SPACE-TIMES}

In this appendix, we obtain the Hamiltonian of the massless scalar field propagating in a general static spherically symmetric space-time. We show that for a particular choice of time slicing such a Hamiltonian reduces to the Hamiltonian of a scalar field in flat space-time.

In Ref. [16], two of the authors (S. D. and S. Sh.) showed that in a fixed Lemaitre time coordinate, the Hamiltonian of the scalar field propagating in Schwarzschild space-time reduces to the scalar field Hamiltonian in flat space-time.
In this appendix, we extend the analysis for any nondegenerate static spherically symmetric space-times.

Let us consider the following line element:

$$
d s^{2}=-A(\tau, \xi) d \tau^{2}+\frac{d \xi^{2}}{B(\tau, \xi)}+\rho^{2}(\tau, \xi) d \Omega^{2}
$$

where $A, B, \rho$ are continuous, differentiable functions of $(\tau, \xi)$ and $d \Omega^{2}=d \theta^{2}+\sin ^{2} \theta d \phi^{2}$ is the metric on the unit 2 -sphere. The action for the scalar field propagating in the above background is given by

$$
\begin{aligned}
S= & -\frac{1}{2} \int d^{4} x \sqrt{-g} g^{\mu \nu} \partial_{\mu} \varphi \partial_{\nu} \varphi \\
= & -\frac{1}{2} \sum_{l m} \int d \tau d \xi\left[-\frac{\rho^{2}}{\sqrt{A B}}\left(\partial_{\tau} \varphi_{l m}\right)^{2}\right. \\
& \left.+\sqrt{A B} \rho^{2}\left(\partial_{\xi} \varphi_{l m}\right)^{2}+l(l+1) \sqrt{\frac{A}{B}} \varphi_{l m}^{2}\right],
\end{aligned}
$$

where we have decomposed $\varphi$ in terms of the real spherical harmonics $\left(Z_{l m}(\theta, \phi)\right)$ :

$$
\varphi\left(x^{\mu}\right)=\sum_{l m} \varphi_{l m}(\tau, \xi) Z_{l m}(\theta, \phi) .
$$

Following the standard rules, the canonical momenta and Hamiltonian of the field are given by

$$
\begin{gathered}
\Pi_{l m}=\frac{\partial \mathcal{L}}{\partial\left(\partial_{\tau} \varphi_{l m}\right)}=\frac{\rho^{2}}{\sqrt{A B}} \partial_{\tau} \varphi_{l m}, \\
H_{l m}(\tau)=\frac{1}{2} \int_{\tau}^{\infty} d \xi\left[\frac{\sqrt{A B}}{\rho^{2}} \Pi_{l m}^{2}+\sqrt{A B} \rho^{2}\left(\partial_{\xi} \varphi_{l m}\right)^{2}\right. \\
\left.+l(l+1) \sqrt{\frac{A}{B}} \varphi_{l m}^{2}\right], \\
H=\sum_{l m} H_{l m} .
\end{gathered}
$$

The canonical variables $\left(\varphi_{l m}, \Pi_{l m}\right)$ satisfy the Poisson brackets

$$
\begin{aligned}
\left\{\varphi_{l m}(\tau, \xi), \Pi_{l m}\left(\tau, \xi^{\prime}\right)\right\} & =\delta\left(\xi-\xi^{\prime}\right), \\
\left\{\varphi_{l m}(\tau, \xi), \varphi_{l m}\left(\tau, \xi^{\prime}\right)\right\} & =0=\left\{\Pi_{l m}(\tau, \xi), \Pi_{l m}\left(\tau, \xi^{\prime}\right)\right\}
\end{aligned}
$$

Having obtained the general Hamiltonian, our next step is to show that this reduces to the flat space-time Hamiltonian of the scalar field in a fixed Lemaître time. In the timedependent Lemaitre coordinates [22,26] the line element is given by (B1) with

$$
A(\tau, \xi)=1, \quad B(\tau, \xi)=\frac{1}{1-f(r)}, \quad \rho(\tau, \xi)=r,
$$

where $r=r(\tau, \xi)$.

The line element in Lemaitre coordinates is related to that in the time-independent Schwarzschild coordinates, 


$$
\begin{aligned}
& \text { viz., } \\
& \qquad d s^{2}=-f(r) d t^{2}+\frac{d r}{f(r)}+r^{2} d \Omega^{2}, \quad f\left(r=r_{h}\right)=0
\end{aligned}
$$

by the following transformation relations [26]:

$$
\begin{aligned}
\tau & =t \pm \int d r \frac{\sqrt{1-f(r)}}{f(r)}, \\
\xi & =t+\int d r \frac{[1-f(r)]^{-1 / 2}}{f(r)}, \\
\xi-\tau & =\int \frac{d r}{\sqrt{1-f(r)}} .
\end{aligned}
$$

The advantage of the Lemaitre coordinate over the Schwarzschild coordinate is that (i) the former is not singular at the horizon $r_{h}$ as opposed to the latter, and (ii) $\xi$ (or, $\tau$ ) are space- (or, time)-like everywhere while $r$ (or, $t$ ) is space- (or, time)-like only for $r>r_{h}$.

Substituting the relations (B7) in the general Hamiltonian (B5), we get

$$
\begin{aligned}
H_{l m}(\tau)= & \frac{1}{2} \int_{\tau}^{\infty} d \xi\left[\frac{1}{r^{2} \sqrt{1-f(r)}} \Pi_{l m}^{2}+\frac{r^{2}}{\sqrt{1-f(r)}}\right. \\
& \left.\times\left(\partial_{\xi} \varphi_{l m}\right)^{2}+l(l+1) \sqrt{1-f(r)} \varphi_{l m}^{2}\right]
\end{aligned}
$$

where the conjugate variables satisfy the Poisson brackets (B6). Note that the scalar field and the Hamiltonian depend explicitly on the Lemaitre time.

Next, choosing the Lemaittre time $\left(\tau=\tau_{0}=0\right)$, the relations (B9) lead to

$$
\frac{d \xi}{d r}=\frac{1}{\sqrt{1-f(r)}} .
$$

If we set $d \theta=d \phi=0$, then for the fixed Lemaitre time $\tau_{0}$ it follows that $d s^{2}=d \xi^{2} / B\left(\tau_{0}, \xi\right)=d r^{2}$, i.e., the covariant cutoff is $|d s|=d r$.

Substituting the above relation (B11) in the Hamiltonian (B10) we get

$H_{l m}(0)=\frac{1}{2} \int_{0}^{\infty} d r\left[\frac{\Pi_{l m}^{2} r^{-2}}{1-f(r)}+r^{2}\left(\partial_{r} \varphi_{l m}\right)^{2}+l(l+1) \varphi_{l m}^{2}\right]$

where the variables $\left(\varphi_{l m}, \Pi_{l m}\right)$ satisfy the relation

$$
\left\{\varphi_{l m}(r), \Pi_{l m}\left(r^{\prime}\right)\right\}=\sqrt{1-f(r)} \delta\left(r-r^{\prime}\right) .
$$

Performing the following canonical transformations:

$$
\Pi_{l m} \rightarrow r \sqrt{1-f(r)} \Pi_{l m}, \quad \varphi_{l m} \rightarrow \frac{\varphi_{l m}}{r}
$$

one obtains [27]

$$
\begin{aligned}
H= & \sum_{l m} \frac{1}{2} \int_{0}^{\infty} d r\left\{\pi_{l m}^{2}(r)+r^{2}\left[\frac{\partial}{\partial r}\left(\frac{\varphi_{l m}(r)}{r}\right)\right]^{2}\right. \\
& \left.+\frac{l(l+1)}{r^{2}} \varphi_{l m}^{2}(r)\right\} .
\end{aligned}
$$

This is nothing but the Hamiltonian of a free scalar field propagating in flat space-time. This is true for any fixed value of $\tau$, provided the scalar field is traced over either the region $r \in\left(0, r_{h}\right]$ or the region $r \in\left[r_{h}, \infty\right)$. Note that the black-hole singularity can be entirely avoided for the latter choice. Now for evaluating time-independent quantities such as entropy, it suffices to use the above Hamiltonian (the same cannot be said for time-dependent quantities).

The approach here differs from that of Ref. [28] where the authors divide the exterior region $r \geq r_{s}$ into two by introducing an hypothetical spherical surface and obtain the entanglement entropy of that surface. In contrast, we consider the complete $r \geq r_{s}$ region and obtain the entropy for the BH horizon. We discuss the possible extensions in Sec. VI.

\section{APPENDIX C: ENTANGLEMENT ENTROPY FOR GS AND ES}

For the sake of completeness, we outline the essential steps in the computation of entanglement entropy for ground state and first-excited state. In the following, we denote all the quantities, viz., wave function, density matrix, etc. by the symbol/suffix 0 for GS and by 1 for the first ES.

\section{Ground state}

In this case the wave function (10) reduces to (on setting $\nu_{i}=0$, for all $i$ ):

$$
\begin{aligned}
\psi_{0}\left(x_{1}, \ldots, x_{N}\right) & =\prod_{i=1}^{N} \mathcal{N}_{i}^{(0)} \exp \left(-\frac{1}{2} k_{D i}^{1 / 2} \underline{\mathbf{x}}_{i}^{2}\right) \\
& =\left(\frac{|\Omega|}{\pi^{N}}\right)^{1 / 4} \exp \left[-\frac{x^{T} \Omega x}{2}\right]
\end{aligned}
$$

Let us decompose

$$
\Omega=\left(\begin{array}{cc}
A & B \\
B^{T} & C
\end{array}\right)
$$

and define

$$
\beta=\frac{B^{T} A^{-1} B}{2}, \quad \gamma=C-\beta,
$$

where $A$ is an $n \times n$ symmetric matrix, $B$ is an $n \times(N-$ $n)$ matrix, and $C, \beta, \gamma$ are all $(N-n) \times(N-n)$ symmetric matrices. The density matrix (12) reduces to [7] 


$$
\rho_{0}\left(t ; t^{\prime}\right)=\sqrt{\frac{|\Omega|}{\pi^{N-n}|A|}} \exp \left[-\frac{t^{T} \gamma t+t^{\prime T} \gamma t^{\prime}}{2}+t^{T} \beta t^{\prime}\right] .
$$

In the above, the matrices $B$ and $\beta$ are nonzero if and only if the HOs are interacting. Because of the Gaussian nature of $\rho_{0}\left(t ; t^{\prime}\right)(\mathrm{C} 4)$, one can make a series of unitary transformations:

$$
\begin{aligned}
& V \gamma V^{T}=\gamma_{D}=\operatorname{diag}, \quad \bar{\beta} \equiv \gamma_{D}^{-1 / 2} V \beta V^{T} \gamma_{D}^{-1 / 2}, \\
& W \bar{\beta} W^{T}=\bar{\beta}_{D}=\operatorname{diag}, \quad v \equiv W^{T} \gamma_{D}^{1 / 2} V,
\end{aligned}
$$

such that it reduces to a product of $(N-n)$, two HO $(N=$ $2)$ density matrices, in each of which one oscillator $(n=1)$ is traced over [7]:

$$
\rho_{0}\left(t ; t^{\prime}\right)=\sqrt{\frac{|\Omega|}{\pi^{N-n}|A|}} \exp \left[-\frac{t^{T} \gamma t+t^{\prime T} \gamma t^{\prime}}{2}+t^{T} \beta t^{\prime}\right] .
$$

In the above, the matrices $B$ and $\beta$ are nonzero if and only if the HOs are interacting. Because of the Gaussian nature of $\rho_{0}\left(t ; t^{\prime}\right)(\mathrm{C} 4)$, one can make a series of unitary transformations:

$$
\begin{gathered}
V \gamma V^{T}=\gamma_{D}=\operatorname{diag}, \quad \bar{\beta} \equiv \gamma_{D}^{-1 / 2} V \beta V^{T} \gamma_{D}^{-1 / 2}, \\
W \bar{\beta} W^{T}=\bar{\beta}_{D}=\operatorname{diag}, \quad v \equiv W^{T} \gamma_{D}^{1 / 2} V,
\end{gathered}
$$

such that it reduces to a product of $(N-n)$, two $\mathrm{HO}(N=$ $2)$ density matrices, in each of which one oscillator $(n=1)$ is traced over [7]:

$$
\rho_{0}\left(t ; t^{\prime}\right)=\sqrt{\frac{|\Omega|}{\pi^{N-n}|A|}} \prod_{i=1}^{N-n} \exp \left[-\frac{v_{i}^{2}+v_{i}^{\prime 2}}{2}+\bar{\beta}_{i} v_{i} \boldsymbol{v}_{i}^{\prime}\right],
$$

where $v_{i} \in v$ and $\bar{\beta}_{i} \in \bar{\beta}$. The corresponding entropy is a sum of two $\mathrm{HO}$ entropies [7]:

$$
\begin{aligned}
S & =-\sum_{i=1}^{N-n} \ln \left[1-\xi_{i}\right]+\frac{\xi_{i}}{1-\xi_{i}} \ln \xi_{i} \\
\xi_{i} & =\frac{\bar{\beta}_{i}}{1+\sqrt{1-\bar{\beta}_{i}^{2}}} .
\end{aligned}
$$

For the total Hamiltonian $H$, the entanglement entropy is

$$
S=\sum_{l=0}^{l_{\max }}(2 l+1) S_{l}=0.3\left(\frac{R}{a}\right)^{2},
$$

where $(2 l+1)$ is the degeneracy factor that follows from the spherical symmetry of the Hamiltonian, $R=$ $a(n+0.5)$ is the radius of the hypothetical spherical surface - the horizon - the DOF inside of which are traced over, and $S_{l}$ is the entropy for a given $l$. Although ideally the upper limit $l_{\max }$ should be infinity, for numerical esti- mation of the entropy (for a certain precision) a very large value of $l_{\max }$ is assigned in practice. The precision goal $\mathrm{Pr}$ is set by demanding that the maximum value of $l \equiv l_{\max }$ should be such that the percentage change in entropy

$$
\frac{S\left(l_{\max }\right)-S\left(l_{\max }-5\right)}{S\left(l_{\max }-5\right)} \times 100<P r .
$$

For instance, if $\operatorname{Pr}=0.01$, the numerical error in the total entropy estimation is less than $0.01 \%$.

In the cases of GCS and SS, it has been shown in [14] that the expression for the total entropy is the same (up to irrelevant multiplicative factors) as that for GS.

\section{First excited state}

In this case, we consider one $\mathrm{HO}$ is in the excited state while the rest $N-1$ are in their GS [13,14]. From Eq. (10), we have

$$
\begin{aligned}
\psi_{1}\left(x_{1} \ldots x_{N}\right)= & \sum_{i=1}^{N}\left(\frac{k_{D i}}{4 \pi}\right)^{1 / 4} \alpha_{i} \mathcal{H}_{1}\left(k_{D i}^{1 / 4} x_{i} \underline{\mathrm{x}}_{i}\right) \\
& \times \exp \left(-\frac{1}{2} \sum_{j} k_{D j}^{1 / 2} \underline{\mathrm{x}}_{j}^{2}\right)
\end{aligned}
$$

In terms of the pure GS wave function (C1) this can be written as

$$
\psi_{1}\left(x_{1} \ldots x_{N}\right)=\sqrt{2}\left(\alpha^{T} K_{D}^{1 / 2} \underline{x}\right) \psi_{0}\left(x_{1}, \ldots, x_{N}\right),
$$

where

$$
\alpha^{T}=\left(\alpha_{1}, \ldots, \alpha_{N}\right)
$$

are the expansion coefficients and the normalization of $\psi_{1}$ requires $\alpha^{T} \alpha=1$.

Using Eq. (12) the density matrix can be evaluated and is given by

$$
\begin{aligned}
\rho_{1}\left(t ; t^{\prime}\right) & =2 \int \prod_{i=1}^{n} d x_{i}\left[x^{\prime T} \Lambda x\right] \psi_{0}\left(x_{i} ; t\right) \psi_{0}^{\star}\left(x_{i} ; t^{\prime}\right) \\
& =\kappa\left[1-\frac{t^{T} \Lambda_{\gamma} t+t^{\prime T} \Lambda_{\gamma} t^{\prime}}{2}+t^{T} \Lambda_{\beta} t^{\prime}\right] \rho_{0}\left(t ; t^{\prime}\right)
\end{aligned}
$$

where $\Lambda$ is a $N \times N$ matrix defined by

$$
\Lambda=U^{T} K_{D}^{1 / 4} \alpha \alpha^{T} K_{D}^{1 / 4} U \equiv\left(\begin{array}{cc}
\Lambda_{A} & \Lambda_{B} \\
\Lambda_{B}^{T} & \Lambda_{C}
\end{array}\right)
$$

$\Lambda_{A}$ is an $n \times n$ symmetric matrix, $\Lambda_{B}$ is an $n \times(N-n)$ matrix, $\Lambda_{C}$ is an $(N-n) \times(N-n)$ symmetric matrix, and $\kappa=\operatorname{Tr}\left(\Lambda_{A} A^{-1}\right)$. The $(N-n) \times(N-n)$ matrices $\Lambda_{\beta}$ and $\Lambda_{\gamma}$ are given by 


$$
\begin{aligned}
\Lambda_{\beta}= & \frac{1}{\kappa}\left(2 \Lambda_{C}-\Lambda_{B}^{T} A^{-1} B-B^{T} A^{-1} \Lambda_{B}\right. \\
& \left.+B^{T} A^{-1} \Lambda_{A} A^{-1} B\right), \\
\Lambda_{\gamma}= & \frac{1}{\kappa}\left(2 \Lambda_{B}^{T} A^{-1} B-B^{T} A^{-1} \Lambda_{A} A^{-1} B\right) .
\end{aligned}
$$

$\Lambda_{\beta}$ is symmetric, while $\Lambda_{\gamma}$ is not necessarily symmetric due to the presence of the first term on the right-hand side.

In general, unlike the GS density matrix $\rho_{0}(\mathrm{C} 4)$, the ES density matrix $\rho_{1}(\mathrm{C} 15)$ cannot be factorized into $(N-n)$ two HO density matrices. However, $\rho_{0}$ is a Gaussian that attenuates virtually to zero beyond its few sigma limits. Therefore, if

$$
\epsilon_{1} \equiv t_{\max }^{T} \Lambda_{\beta} t_{\max } \ll 1, \quad \epsilon_{2} \equiv t_{\max }^{T} \Lambda_{\gamma} t_{\max } \ll 1
$$

where

$$
t_{\max }^{T}=\left(\frac{3(N-n)}{\sqrt{2 \operatorname{Tr}(\gamma-\beta)}}\right)(1,1, \ldots)
$$

corresponding to $3 \sigma$ limits of the Gaussian inside $\rho_{0}$, then one may approximate

$$
\begin{aligned}
1 & -\frac{t^{T} \Lambda_{\gamma} t+t^{\prime T} \Lambda_{\gamma} t^{\prime}}{2}+t^{T} \Lambda_{\beta} t^{\prime} \\
& \approx \exp \left[-\frac{t^{T} \Lambda_{\gamma} t+t^{\prime T} \Lambda_{\gamma} t^{\prime}}{2}+t^{T} \Lambda_{\beta} t^{\prime}\right] .
\end{aligned}
$$

As such, with the following shift of parameters,

$$
\beta^{\prime} \equiv \beta+\Lambda_{\beta}, \quad \gamma^{\prime} \equiv \gamma+\Lambda_{\gamma}
$$

the approximated ES density matrix is also a Gaussian,

$$
\rho_{1}\left(t ; t^{\prime}\right) \approx \kappa \exp \left[-\frac{t^{T} \gamma^{\prime} t+t^{\prime T} \gamma^{\prime} t^{\prime}}{2}+t^{T} \beta^{\prime} t^{\prime}\right]
$$

This can be factorized once again into two HO density matrices, and the associated entanglement entropy can be computed. For the following set of values: $N=300, n=$ $100-200, o=10-50$, $o$ being the number of last nonvanishing entries in the vector $\alpha^{T}$, i.e., $\alpha^{T}=(1 / \sqrt{o}) \times$ $(0, \cdots, 0,1, \cdots, 1)$, the entropy computation is done numerically (using MATLAB) in [14]. The precision setting in the computation had been $0.1 \%$ and the criteria $(\mathrm{C} 18)$ is satisfied for the above choice of the parameters $N, n$, and $o$. The results show that the ES entropy scales as a power of the area. The power, however, is always less than unity (for any $o>0$ ) and is lesser and lesser, the higher the value of $o$. The AL is thus always violated for the chosen range of values of $n(=100-200)$. The other interesting observation made in [14] is the shifting of peaks in the variation of the partial waves $(2 l+1) S_{l}$ with $l$ for ES as compared to the case for GS.
[1] J.D. Bekenstein, Lett. Nuovo Cimento 4, 737 (1972); Phys. Rev. D 7, 2333 (1973); Phys. Rev. D 9, 3292 (1974); Phys. Rev. D 12, 3077 (1975).

[2] S. W. Hawking, Nature (London) 248, 30 (1974); Commun. Math. Phys. 43, 199 (1975).

[3] R. M. Wald, Living Rev. Relativity 4, 6 (2001).

[4] W. Israel, Lect. Notes Phys. 617, 15 (2003); D. N. Page, New J. Phys. 7, 203 (2005).

[5] A. Strominger and C. Vafa, Phys. Lett. B 379, 99 (1996); A. Ashtekar, J. Baez, A. Corichi, and K. Krasnov, Phys. Rev. Lett. 80, 904 (1998); S. Carlip, ibid. 88, 241301 (2002); A. Dasgupta, Classical Quantum Gravity 23, 635 (2006).

[6] L. Bombelli, R. K. Koul, J. Lee, and R. Sorkin, Phys. Rev. D 34, 373 (1986).

[7] M. Srednicki, Phys. Rev. Lett. 71, 666 (1993).

[8] G. 't Hooft, Nucl. Phys. B256, 727 (1985); V. P. Frolov and D. V. Fursaev, Phys. Rev. D 56, 2212 (1997).

[9] J. D. Bekenstein, arXiv:gr-qc/9409015.

[10] M. B. Plenio, J. Eisert, J. Dreissig, and M. Cramer, Phys. Rev. Lett. 94, 060503 (2005); M. Cramer, J. Eisert, M. B. Plenio, and J. Dreissig, Phys. Rev. A 73, 012309 (2006).

[11] R. Brustein and A. Yarom, Nucl. Phys. B709, 391 (2005); R. Brustein et al., J. High Energy Phys. 01 (2006) 098; J.
High Energy Phys. 04 (2007) 086; D. Marolf and A. Yarom, J. High Energy Phys. 01 (2006) 141;

[12] S. Ryu and T. Takayanagi, Phys. Rev. Lett. 96, 181602 (2006); J. High Energy Phys. 08 (2006) 045.

[13] M. Ahmadi, S. Das, and S. Shankaranarayanan, Can. J. Phys. 84, 493 (2006); S. Das and S. Shankaranarayanan, J. Phys. Conf. Ser. 68, 012015 (2007).

[14] S. Das and S. Shankaranarayanan, Phys. Rev. D 73, 121701 (2006).

[15] R. M. Wald, Phys. Rev. D 48, R3427 (1993).

[16] S. Das and S. Shankaranarayanan, Classical Quantum Gravity 24, 5299 (2007); and arXiv:0708.2098.

[17] See for example A. Erdelyi, Asymptoic Analysis (Dover Publications, New York, 1956); G. H. Hardy and E. M. Wright, An Introduction to the Theory of Numbers (Clarendon Press, Oxford, 1975); N.G. de Bruijn, Asymptotic Methods in Analysis (Dover Publications, New York, 1981); for a general definition of asymptotic equivalence see also http://thesaurus.maths.org/.

[18] T. Jacobson and R. C. Myers, Phys. Rev. Lett. 70, 3684 (1993); R. C. Myers and J.Z. Simon, Phys. Rev. D 38, 2434 (1988); A. Paranjape, S. Sarkar, and T. Padmanabhan, Phys. Rev. D 74, 104015 (2006).

[19] S. Das, S. Shankaranarayanan, and S. Sur (work in 
progress).

[20] S. Sarkar, S. Shankaranarayanan, and L. Sriramkumar, arXiv:0710.2013.

[21] G. 't Hooft and M.J.G. Veltman, Ann. Inst. Henri Poincaré, A 20, 69 (1974); N.H. Barth and S.M. Christensen, Phys. Rev. D 28, 1876 (1983).

[22] L.D. Landau and E. M. Lifshitz, Classical Theory of Fields, Course of Theoretical Physics Vol. 2 (Pergamon Press, New York 1975).

[23] See for example S. Chandrasekhar, The Mathematical Theory of Black Holes (Clarendon Press, Oxford, 1992);
K. D. Kokkotas and B. G. Schmidt, Living Rev. Relativity 2, 2 (1999).

[24] H. Kodama and A. Ishibashi, Prog. Theor. Phys. 111, 29 (2004); S. Das and S. Shankaranarayanan, Classical Quantum Gravity 22, L7 (2005) and references therein.

[25] F. J. Zerelli, Phys. Rev. D 2, 2141 (1970); V. Moncrief, Ann. Phys. (N.Y.) 88, 323 (1974).

[26] S. Shankaranarayanan, Phys. Rev. D 67, 084026 (2003).

[27] K. Melnikov and M. Weinstein, Int. J. Mod. Phys. D 13, 1595 (2004).

[28] S. Mukohyama et al., Phys. Rev. D 58, 064001 (1998). 